Voix et Images

voixetimages

\title{
L'écriture comme appropriation de soi et du monde
}

\section{Michel Nareau et Jacques Pelletier}

Volume 41, numéro 1 (121), automne 2015

Louis Hamelin

URI : https://id.erudit.org/iderudit/1033954ar

DOI : https://doi.org/10.7202/1033954ar

Aller au sommaire du numéro

Éditeur(s)

Université du Québec à Montréal

ISSN

0318-9201 (imprimé)

1705-933X (numérique)

Découvrir la revue

Citer ce document

Nareau, M. \& Pelletier, J. (2015). L'écriture comme appropriation de soi et du monde. Voix et Images, 41(1), 7-11. https://doi.org/10.7202/1033954ar d'utilisation que vous pouvez consulter en ligne.

https://apropos.erudit.org/fr/usagers/politique-dutilisation/ 


\title{
L'ÉCRITURE COMME APPROPRIATION DE SOI ET D U MONDE
}

\author{
$+++$ \\ MICHEL NAREAU \\ Figura-Université du Québec à Montréal \\ JACQUES PELLETIER \\ Université du Québec à Montréal
}

Louis Hamelin, avec La rage ${ }^{1}$, a surgi comme un météorite dans le firmament littéraire québécois au tournant de la décennie 1990, provoquant un effet analogue à celui produit vingt-cinq ans plus tôt par la publication d'Une saison dans la vie d'Emmanuel de Marie-Claire Blais, de Prochain épisode d'Hubert Aquin ou de L'avalée des avalés de Réjean Ducharme. Son roman apparaissait d'une certaine manière comme un prolongement de ces œuvres phares, tout en s'avérant radicalement nouveau sur le plan proprement stylistique. Rapprochée également des œuvres d'un Jacques Ferron ou d'un Victor-Lévy Beaulieu, son entreprise était reçue comme un événement exceptionnel témoignant d'une nouvelle sensibilité culturelle, sociale et politique, propre à la génération $\mathrm{X}$, marquée par le doute, voire le désespoir, lié à l'effondrement des grandes espérances associées aux Trente Glorieuses, lesquelles furent suivies par la montée et la domination du nouveau conservatisme caractéristique de l'ère Reagan/ Thatcher qui sévit toujours aujourd'hui.

Le héros narrateur de La rage, de même que les amis et camarades qui l'entourent, symbolisent le destin d'une génération promise à la précarité et à la marginalité, et qui porte, du coup, un regard désabusé sur le monde dans lequel elle est appelée à vivoter. En cela, ces personnages d'antihéros apparaissent expropriés de la vie et de l'espoir comme, sur un plan plus général, les habitants de Sainte-Scholastique et de la plaine laurentienne sont dépossédés au même moment de leurs terres fertiles au profit de l'aéroport de Mirabel. Cet éléphant blanc, cette cathédrale du vide et du néant, incarne et synthétise dans le roman, avec les centres commerciaux des banlieues tentaculaires qui ceinturent Montréal, toute l'horreur climatisée et aseptisée du monde contemporain. Le refus de cet univers cauchemardesque prend la forme d'un mal de vivre aigu et lancinant, pouvant aller jusqu'au suicide, au meurtre, voire à l'action terroriste. L'assaut lancé par le héros, poussé par la rage, contre la tour de contrôle de Mirabel l'illustre dramatiquement dans l'épisode de fureur, en forme d'«envolée des envolées», qui met fin au roman de manière flamboyante, en faisant un clin d'œil au récit mythique de Réjean Ducharme.

1 Pour les références complètes aux œuvres d'Hamelin, le lecteur pourra consulter la bibliographie préparée par Laurence Perron, dans le présent dossier, p. 101-119. 
Élaborée au temps de la genèse de l'œuvre, la problématique de la désappropriation de soi et du monde sera reprise et approfondie dans les récits qui vont succéder à La rage, culminant dans cet immense roman que constitue La constellation du Lynx. Cette fresque au souffle épique s'offre en effet comme l'accomplissement en acte du "Grand Roman Québécois» fantasmé dans Ces spectres agités, idéal qui inspire l'écrivain depuis les tout débuts de son entreprise.

Comme c'était le cas pour La rage, on rencontre dans cette dernière œuvre l'imbrication serrée de ce que l'auteur, invoquant l'exemple du Roi des Aulnes de Michel Tournier, conçoit, dans son essai L'humain isolé, comme «trois grandes couches de sens: l'Histoire, le Mythe, le Destin ${ }^{2} »$. L'Histoire, en l'occurrence la crise d'Octobre 1970. Le Mythe, celui de la victime expiatoire ou du bouc émissaire, le lynx sacrifié remplaçant le renard ailé et rageur du premier roman. Le Destin, enfin, d'un groupe de jeunes gens rebelles et manipulés, ou du moins manipulables, émergeant de ce lieu trouble et mythique à sa manière qu'est Ville Jacques-Cartier et d'un peuple à la dérive dans un monde de bruit et de fureur, opaque et menaçant, dominé par les agents doubles qui en tirent les ficelles dans l'ombre. Cette crise fameuse demeure encore aujourd'hui, on le sait, un mystère irrésolu, du moins dans sa dimension factuelle et événementielle, qui paraît s'épaissir à mesure que s'accumulent à son propos les commissions d'enquête, les travaux des historiens, et que se multiplient les témoignages des acteurs et des témoins, victimes d'étonnants trous de mémoire.

La fiction pourrait-elle faire mieux que les recherches expertes et pointues? C'est le pari audacieux du romancier, qui utilise l'imagination comme «instrument d'investigation historique", ainsi qu'il le signale lui-même. Il suggère du même souffle que la version qu'il donne de la crise d'Octobre dans sa fiction est plus «vraie», c'està-dire plus cohérente et plausible, que la version officielle proposée tant par les militants du Front de libération du Québec (FLQ) que par la police et les pouvoirs publics. La réinterprétation qu'il propose passe par la voie d'une sorte de polar politique, qui emprunte et excède à la fois les règles et les codes du genre, et par celle d'un roman à clefs et à énigmes, doublé d'une fresque historique qui rappelle le contexte social et politique immédiat et lointain dans lequel prend place cette histoire, perspective ample qui permet de mieux comprendre les comportements des acteurs, aussi bien les masqués que ceux qui se présentent à découvert.

Sur le plan compositionnel, architectonique, La constellation du Lynx se présente comme un roman polyphonique, faisant entendre plusieurs voix et autant de points de vue. Et cela dans le cadre d'une courtepointe rassemblant des fragments évoquant de multiples espaces et temporalités réunis autour de l'épicentre que constitue la crise d'Octobre. Chaque fragment s'offre à la fois comme une énigme et un révélateur, favorisant le rebondissement du récit, qui interroge et déconstruit l'Histoire officielle tout en entraînant les lecteurs dans une enquête palpitante, à l'instar des polars les plus réussis.

Le roman de Louis Hamelin dépasse toutefois les limites habituelles du récit policier, qu'il intègre dans une réflexion plus vaste sur le devenir de la société québécoise contemporaine. En cela, il s'offre comme une «œuvre fondatrice», un

2 Louis Hamelin, L’humain isolé, Trois-Pistoles, Éditions Trois-Pistoles, coll. «Écrire», 2006, p. 25. 
accomplissement dont la portée culturelle, sociale et politique est incontestable, roman kaléidoscopique dans lequel on peut voir, comme dans un miroir grossissant, ce que nous sommes et ce que nous devenons comme peuple dans une Histoire embrouillée, où nous peinons à reconnaître notre vérité et à tracer notre destin.

Dans son dernier ouvrage, Fabrications. Essai sur la fiction et l'histoire, l'auteur revient sur cette expérience d'écriture qui l'a mobilisé durant une décennie. Dans cet essai que l'on pourrait qualifier de narratif, et qui est composé comme une mosaïque dédoublant, sur le plan formel, le propos théorique exposé, il nous donne à voir en quelque sorte le making of de son ambitieux roman, nourri des témoignages des acteurs, des documents d'archives, des récits historiques aussi bien que de la lecture d'écrivains se confrontant, comme lui-même, à l'Histoire, notamment Léon Tolstoï et Norman Mailer. Qu'est-ce que l'Histoire et comment la narre-t-on? S'agit-il d'abord d'une affaire d'individus ou de masses? Se déroulant au grand jour ou se déployant dans le secret? La raconte-t-on en privilégiant un regard ou plusieurs points de vue? $\mathrm{Et}$, dans le dernier cas, comment les orchestre-t-on?

La production romanesque de Louis Hamelin s'inscrit entre les deux bornes que représentent la réussite originaire que demeure La rage et l'œuvre de maturité qu'incarne La constellation du Lynx et qui en constitue le terme provisoire. Il serait trop long de la décrire en détail ici. On se contentera donc de rappeler, pour mémoire, que la problématique générale de la dépossession et de son versant positif, la réappropriation, qui la traverse d'un bout à l'autre se cristallise dans un certain nombre de thèmes et de préoccupations. On retrouve ainsi, entre ses divers romans, par-delà leurs manifestations singulières et leurs différences réelles, une cohérence qui assure leur profonde unité. Elle repose pour l'essentiel sur une critique acerbe, bien qu'exercée le plus souvent sur le mode ironique, de la dégradation à laquelle conduit la logique de l'expropriation et de la domination du monde vivant, de la nature aussi bien que des rapports sociaux. C'est à cette logique mortifère que s'opposent, directement ou de manière oblique, les héros de Louis Hamelin, soucieux d'environnement et en cela créatures conséquentes d'un écrivain venu à la littérature après un long détour par la science. Le premier livre auquel il a travaillé, confie-t-il dans L'humain isolé, était «un ouvrage d'histoire naturelle ${ }^{3}$ » dont les romans comme La rage ou Le soleil des gouffres apparaissent d'une certaine manière comme des prolongements fictionnels. L'empreinte écologique remonte en l'occurrence à très loin: il s'agit d'une préoccupation précoce et qui semble établie à demeure comme le fait bien voir, entre autres, la figure du lynx, chasseur et chassé, prédateur et proie, dans son dernier roman.

Sur le plan de l'écriture, on sait que cette œuvre se distingue, c'est l'une de ses constantes, par une intertextualité foisonnante, ramifiée, parfois latente, parfois affichée de manière plus ostentatoire. Celle-ci se manifeste à travers l'évocation de plusieurs grandes figures de la littérature québécoise et mondiale, allant d'Aquin, de Ducharme, de Jack Kerouac, dont les ombres se profilent dans La rage, à Ferron et à Mario Vargas Llosa dans La constellation du Lynx en passant par bien d'autres, dont Carlos Fuentes métamorphosé en Charles Dessources dans un passage désopilant qui

3 Ibid., p. 31. 
raconte les problèmes d'incontinence et d'hémorroïdes du grand homme dans Le soleil des gouffres!

La posture de Louis Hamelin à l'endroit de ses maîtres en écriture relève donc d'une sorte de respect ironique: admiration réelle pour leur grandeur, mais sans obséquiosité, s'apparentant parfois à une distance moqueuse. Tout se passe comme s'il s'agissait davantage de confrères, avec lesquels on peut blaguer, que de figures d'autorité, comme si la dette à leur endroit, une fois reconnue, était aussitôt acquittée. Et elle l'est, non seulement dans les grandes références auxquelles renvoient les romans, mais aussi dans les confidences et aveux très explicites contenus dans L'humain isolé, Le voyage en pot et Fabrications où sont convoquées les figures internationales de James Joyce, de Franz Kafka, de Malcolm Lowry, de Mailer, de Tolstoï ou de Tournier, et québécoises d'Aquin, de Ducharme, de Mordecai Richler et de Gabrielle Roy, mais également de Patrice Desbiens, de Christian Mistral et d'autres personnages qui constituent pour lui une sorte de «marginocratie», une communauté de «frères » élus qu'il semble placer au-dessus de tout et de tous et dont ses personnages de fiction s'avèrent souvent des doublets.

La production de Louis Hamelin est certes diversifiée, mais elle s'élabore à partir de certains traits communs, qui sont explorés par les collaborateurs du dossier: insertion dans des lieux problématiques, reconfiguration des enjeux identitaires, prise en compte d'un imaginaire amérindien, écriture de l'histoire, etc. Les deux premiers textes abordent chacun un pan de cette constance thématique et, à partir d'œuvres spécifiques, ils mettent en lumière certains leitmotive qui traversent l'œuvre. Julien Desrochers montre ainsi que la cartographie des rapports de pouvoir dans La rage oppose une verticalité technologique, représentée par les jumbo jets et la tour de contrôle et qui attise la convoitise, à une horizontalité historique, liée au monde agraire et à ce qui précède l'expropriation de Mirabel. La trajectoire d'Édouard Malarmé, comme celle des autres protagonistes d'Hamelin, viserait à l'inscrire dans cette logique verticale afin qu'il y acquière une place symbolique, notamment par le recours à l'écriture. Si Desrochers traite l'espace comme le lieu d'un rapport de force, Jimmy Thibault s'intéresse davantage à sa dimension continentale, en dégageant dans quelques textes d'Hamelin une tentative pour sortir du modèle identitaire et pour lui préférer une logique de mises en rapport, de relations. En passant de l'identité à l'entité, les protagonistes s'inscrivent dans une logique de rupture (envers les attributions historiques) et de reconfiguration (en développant de nouveaux liens, souvent issus des marges, jugés plus fructueux).

Les trois autres articles traitent de La constellation du Lynx, preuve supplémentaire de la richesse et de la complexité de ce roman. Daniel Laforest s'intéresse à Ville Jacques-Cartier, pour montrer qu'à la lecture d'une horizontalité du déjà-là, Hamelin oppose une archéologie de l'avant-hier et de la verticalité, où l'inscription de l'histoire des lieux fait surgir une infrastructure pionnière et le parcours marginalisé, mais documenté, d'habitations excentrées, ce que Laforest nomme "les cimetières de l'histoire», rattrapés par la crise d'Octobre. Robert Dion, quant à lui, met en résonance le roman d'Hamelin avec le travail de Carl Leblanc concernant l'enjeu de l'écriture de l'histoire, les deux écrivains tissant leur intrigue à partir d'une posture postmémorielle des événements de 1970. La force du texte de Louis Hamelin, 
dans cette perspective, est de travailler avec la multiplicité des discours, sans tenter, formellement et idéologiquement, d'en restituer une ligne univoque. Au contraire, l'écriture ironique et l'enquête mise en scène auraient pour effet d'exacerber les tensions entre les mémoires et d'ainsi inscrire le caractère herméneutique du roman. Le dernier texte du dossier, celui de François Ouellet, arrime trois enjeux majeurs de cette œuvre: l'écriture, l'amour et le père. En remontant le parcours de Sam Nihilo jusqu'à Sauvages, Ouellet montre que l'enquête sur la crise d'Octobre est aussi une quête, celle de Sam, qui hérite de la recherche entreprise par Branlequeue et la mène à terme, grâce à l'amour, à Marie-Québec. Cette quête, nous dit Ouellet, affiche une résolution nouvelle chez Hamelin en unissant amour et écriture, mais au détriment du pays, relégué au second plan.

Pour terminer cette présentation, forcément elliptique, d'une œuvre qui appelle le commentaire par son ampleur, sa portée et sa complexité, on rappellera la conception de la littérature que propose l'auteur dans L'humain isolé: "La littérature, c'est ce que vous écrivez et que personne d'autre ne peut écrire à votre place. La petite part d'humain que vous transportez et que vous allez faire passer sur le papier. Comme un savant qui isole une souche de microbes. La littérature, c'est $l^{\prime}$ humain isolé. .» Et cet humain isolé, c'est l'anagramme bien sûr de Louis Hamelin, l'homme-écriture.

Ce dossier s'offre comme une première tentative d'exploration collective de cette œuvre foisonnante. Il privilégie certains romans: La rage, Le joueur de flûte, La constellation du Lynx et le recueil de nouvelles Sauvages, et certaines dimensions comme l'écologie, l'identité dans une perspective franco-américaine pleinement assumée, le rapport à l'histoire et à la mémoire, ou encore la quête amoureuse qui traverse l'œuvre d'un bout à l'autre. Il ne couvre donc pas tout, ni l'ensemble de cette production ni ses multiples préoccupations. Mais il en dresse tout de même un portrait qui met en lumière quelques-unes de ses composantes centrales et de ses principales orientations, et qui donnera peut-être à d'autres le goût d'en explorer de nouvelles; en cela ce dossier est loin d'être fermé, il appelle au contraire à de nouvelles expéditions dans cette forêt luxuriante.

4 Ibid., p. 100. 